\section{International Scientific Journal Theoretical \& Applied Science}

p-ISSN: 2308-4944 (print) e-ISSN: 2409-0085 (online)

Year: $2016 \quad$ Issue: 9 Volume: 41

Published: $30.09 .2016 \quad$ http://T-Science.org
Sergey Alexandrovich Mishchik Associate Professor, Candidate of Pedagogical Science, Corresponding member of International Academy TAS, Assistant professor Department of Physics, State Maritime University Admiral Ushakov, Russia, sergei_mishik@mail.ru

SECTION 21. Pedagogy. Psychology. Innovation in Education

\title{
DEVELOPMENT OF BENCHMARKS ERTSGAMMING MATHEMATICAL MODELS OF EDUCATIONAL ACTIVITY FORMATIVE RESEARCH EDUCATIONAL FACILITIES
}

\begin{abstract}
The main directions of analysis of development benchmarks ertsgamming mathematical models of learning activities formative research educational facilities relativetional criteria of life, cycling, systematic and phasing, which form a basising cell education space, as well as the use of the twelve pointed star Ertsgam we are on the submission ertsgamming principle which determines the foundations pedagogometric through shaping matrix methods, graph theory and games hyperspace living, psychological and educational activity theory, psychopedagogical system analysis and the theory of the formation of mental actions.

Key words: basicity, formative research, pedagogometric matrix, graphs, ability to live, work, play, cycle, system, phasing, principle ertsgamming, star Ertsgammy.

Language: Russian

Citation: Mishchik SA (2016) DEVELOPMENT OF BENCHMARKS ERTSGAMMING MATHEMATICAL MODELS OF EDUCATIONAL ACTIVITY FORMATIVE RESEARCH EDUCATIONAL FACILITIES. ISJ Theoretical \& Applied Science, 09 (41): 177-182.

Soi: http://s-o-i.org/1.1/TAS-09-41-31 Doi: crossef http://dx.doi.org/10.15863/TAS.2016.09.41.31
\end{abstract}

УДК 372.851

\section{РАЗВИТИЕ БАЗИСНЫХ ПОКАЗАТЕЛЕЙ ЭРЦГАММНОСТИ МАТЕМАТИЧЕСКИХ МОДЕЛЕЙ УЧЕБНОЙ ДЕЯТЕЛЬНОСТИ ФОРМИРУЮЩЕГО ИССЛЕДОВАНИЯ ОБРАЗОВАТЕЛЬНЫХ ОБЪЕКТОВ}

Аннотация: Рассмотрень основные направления анализа развития базисных показателей эригаммности математических моделей учебной деятельности формирующего исследования образовательных объектов относительно критериев жизнедеятельности, ияикличноти, системности и этапности, которые образуют базисную ячейку образовательного пространства, а также применение двенадиати конечной звезды Эригаммы относительно представления принципа эригаммности, который определит основы педагогометрики через формообразование матричными методами, теории графов и игр гиперпространства жизнедеятельности, психолого-педагогической теории деятельности, психологопедагогического системного анализа и теории формирования умственных действий.

Ключевые слова: базисность, формирующее исследование, матричная педагогометрика, графы, жизнедеятельность, деятельность-игра, ијикличность, системность, этапность, принцип эризгаммности, звезда Эригаммы.

\section{Introduction}

Развитие базисных показателей эрцгаммности математических моделей учебной деятельности формирующего исследования образовательных объектов следует рассматривать как реализацию всех основных эрцгаммных объектов образовательного пространства: звезды Эрцгаммы гиперпространства жизнедеятельности
(E1); целостно-системного

цикла жизнедеятельности (Е2); звезды Эрцгаммы системного анализа (Е3); проявления двенадцати этапов и форм познавательного гиперпространства жизнедеятельности относительно образовательного процесса (E4) [1]. Каждый образовательный объект с признаком эрцгаммности, независимо от 
целевого назначения, выполняет собственную функцию психолого-математического представления, имеющего соответствующий показатель целостного развития относительно характеристик собственной собственной значимости. Каждый глобальный объект (E1, E2, E3, E4) образовательного пространства выполняет синфазно три функции: ориентировки, исполнения и контроля каждой фазы развития образовательного процесса. Поэтому можно сформировать матрицу инвариантного состояния образовательного процесса.

\begin{tabular}{|l|l|l|}
\hline$E_{10}$ & $E_{1 и}$ & $E_{1 к}$ \\
\hline$E_{20}$ & $E_{2 и}$ & $E_{2 \kappa}$ \\
\hline$E_{30}$ & $E_{3 и}$ & $E_{3 к}$ \\
\hline$E_{40}$ & $E_{4 и}$ & $E_{4 \text { к }}$ \\
\hline
\end{tabular}

Таблица 1 - Матрица инвариантного состояния образовательного процесса.

\section{Materials and Methods}

Математической моделью целостносистемного цикл жизнедеятельности является образ циклического графа. Пусть X - множество вершин (12) - предметные и деятельностные компоненты, V-множество ребер, соединяющие вершины (12) - время освоения и применения знания. Граф $\mathrm{G}=(\mathrm{X}, \mathrm{V})$ является заданным, если дано множество его вершин $\mathrm{X}$ и способ отображения Г этого множества в самого себя. При этом можно выделить часть ЦСЦЖ и представить её в виде подграфа $\mathrm{G}_{\mathrm{A}}$ графа $\mathrm{G}=(\mathrm{X}$, Г). Если подграф $G_{A}=\left(A, \Gamma_{A}\right)$ целостносистемного цикла имеет лишь часть вершин графа $\mathrm{G}$ и образует пару элементов, то является базисным. Например: (НЦСС) и (ЦСВД) образуют множество А, вместе с дугами, соединяющими эти вершины: $G_{A}=\left(A, \Gamma_{A}\right)$, где

$$
\mathrm{A} \subseteq \mathrm{X}, \quad \Gamma_{\mathrm{A}} \mathrm{X}=(\Gamma \mathrm{x}) \cap \mathrm{A} .
$$

Если учесть, что любой деятельностный компонент имеет три составляющие: ориентировочные, исполнительные и контрольные части действия, то возникает частичный базисный граф $\mathrm{G}_{\Delta}$ по отношению к графу $\mathrm{G}=(\mathrm{X}, \Gamma)$, в котором содержится только часть дуг графа G. Возникают условия: $\mathrm{G}_{\Delta}=(\mathrm{X}, \Delta)$, где $\Delta \mathrm{x} \subseteq \Gamma \mathrm{x}[2]$.

В графологическом представлении целостно-системного цикл жизнедеятельности применяем понятия пути, длины пути и контура. Для описания графа цикла жизнедеятельности используем матрицы смежности и матрицы инцидентности. Представляем временные параметры сетевых графиков ЦСЦЖ и их нахождение; определяем длину критического пути, выявляя событие с нулевыми резервами времени, устанавливаем его топологию. Параметры развёртывания событий ЦСЦЖ исследуются из условий: если $\boldsymbol{t}_{p}(\boldsymbol{i})-$ ранний срок поступления события, то определяется продолжительностью максимального пути, предшествующего этому событию.

$$
t_{p}(i)=\max _{L_{n i}} t\left(L_{n i}\right)
$$

если ј имеет несколько предыдущих событий, то

$$
{ }^{t_{p}} p(j)=\max _{i, j}\left[t_{p}(i)+t(i, j)\right] .
$$

Пусть $t_{\Pi}(i)$ - поздний срок поступления события

$$
t_{\Pi}(i)=t_{k p}-\max t\left(L_{c i}\right),
$$

где $L_{c i}$ - любой путь, следующий за i-м событием, т. е. путь от і-го до завершающего события цепи. Если i имеет несколько последующих путей или событий $\mathrm{j}$, тогда

$$
t_{\Pi}(i)=\min _{i, j}\left[t_{\Pi}(j)-t(i, j)\right] .
$$

Резерв времени на формирование устойчивой структуры ЦСЦЖ определяется из условия: $R(i)=t_{\Pi}(i)-t_{p}(i) \quad$ Данный параметр показывает допустимый период времени на полное представление цикла по задержке наступление этого события, не вызывая увеличение срока выполнения комплекса развития [3].

Процесс принятия решений в условиях определенности при представлении ЦСЦЖ имеет трудность в существовании нескольких критериев, по которым сравниваем исходы. Если имеетя совокупность критериев: , $F_{1}(x), F_{2}(x), \ldots, F_{n}(x), x \in X$, то обобщенный

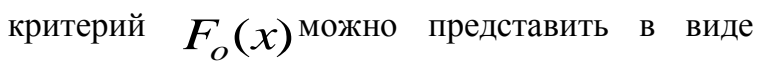
взвешенной суммы 


$$
F_{o}(x)=\sum_{i=1}^{n} W_{i} F_{i}(x)
$$

где $W_{i}$ - вес соответствующего критерия. Тогда определяем

$$
\max _{x} F_{o}(x)
$$

Поэтому задача оптимального представления ЦСЦЖ имеет вид

$$
\max _{x \in X} F_{1}(x)
$$

при ограничениях

$$
\bar{F}_{2}(x) \geq F_{2} \text { доп } . . F_{n}(x) \geq F_{n} \text { доп }
$$

Моделируем анализ ЦСЦЖ принятия решений в условиях риска и неопределенности. Из нескольких критериев для выбора оптимальной стратегии представлений ЦСЦЖ рассматриваем критерии: Вальда (критерий осторожного наблюдателя), который дает гарантированный выигрыш при наихудшем состоянии среды; критерий Гурвица; критерий Лапласа, для которого если неизвестны состояния среды, то все состояния ЦСЦЖ считают равновероятными; критерий Сэвиджа (критерий минимизации сожалений), то есть величине, равной изменению полезности результата развития целостно-системного цикла жизнедеятельности при данном состоянии среды относительно наилучшего возможного процесса воспитания личности.

Любое целостно-системное учебное действие имеет три базисные компонента: ориентировочный, исполнительный и контрольный, которые определяют основные направления математического моделирования ЦСУД. Множество элементов учебного действия можно записать в виде $\grave{A}=\left\{a_{i}\right\}, i=1,2, \ldots, n$, где $\quad a_{i}-i$-й - элемент системного действия, $\mathrm{n}$ - число элементов учебного действия. Каждый элемент ЦСУД характеризуется $m$ конкретными свойствами $z_{1}$, $z_{2}, \ldots, z_{m}$, которые однозначно определяют его в данной системе. Совокупность всех $m$ свойств элемента учебного действия устанавливает его состояние: $z_{i}=\left(z_{i 1}, \ldots, z_{i m}\right)$. Между базисными компонентами ЦСУД существует связь множество зависимостей свойств одного элемента от свойств других элементов системы учебного действия. Множество связей между элементами учебного действия можно представить в виде $Q=\left\{q_{i j}\right\}, i, j=1,2, \ldots, n$. Зависимость свойств элементов учебного действия имеет двусторонний взаимосвязанный характер. Это определяет структуру системы учебного действия - множество элементов системы и связей между ними: $D=\{A, Q\}$ [4].

Структура ЦСУД зависит от статического и динамического состояний. $\mathrm{B}$ условиях статического поведения учебного действия связь между ориентировочным и контрольным компонентами представляется как связь между функциями входа $X(t)$ и выхода $Y(t)$ системы без учета предыдущих ее состояний: $Y(t)=F_{B}[X(t)]$, где $F_{B}-$ функция выходов системы. В условиях динамического состояния учебного действия система зависит не только от функций входов $X(t)$, но и от функций переходов,

$$
Y(t)=F_{B}[X(t), Z(t), z(t-1), \ldots] .
$$

В данном случае можно определить обобщенный показатель качества целостносистемного учебного действия как вектор $Y=\left\{y_{1}, y_{2}, \ldots, y_{n}\right\}$, компоненты которого есть частные показатели отдельных свойств ЦСУД. Размерность $n$ определяется числом системных свойств учебного действия.

При определении обобщенного показателя качества целостно-системного учебного действия будем применять не натуральные частные показатели, а нормированные значения. Это обеспечивает приведение показателей к одному масштабу: $y_{i}^{H O P M}=\frac{y_{i}}{y_{i}^{*}}, \quad i=1,2, \ldots, n$, где $y_{i}^{*}$ - некоторое «идеальное» значение $i$-го показателя ЦСУД. Любое целостно-системное учебное действие можно принять за идеальную систему, если её гипотетическая модель, удовлетворяет всем критериям качества: $Y^{*}=\left\{y_{1}^{*}, y_{2}^{*}, \ldots, y_{3}^{*}\right\}$. Тогда выделяется область адекватности ЦСУД окрестность значений показателей целостносистемных свойств учебного действия. Радиус адекватности имеет нормированное значение, которое определяется зависимостью: $\delta \subseteq \frac{\left|Y^{\partial o n}-Y^{*}\right|}{\left|Y^{*}\right|}$.

Все критерии качества целостно-системного учебного действия определяются тремя типами:

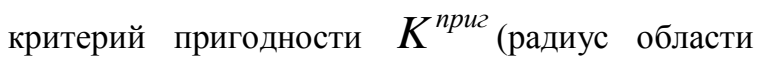
адекватности $\delta$ соответствует допустимым значениям всех частных показателей); критерий оптимальности $K^{\text {опm }}$ (существует хотя бы один частный показатель качества $y_{i}^{j}$, значения которого принадлежат области адекватности с оптимальным радиусом $\delta^{\text {onm }}=0$ ) и критерий 
превосходства $K^{\text {прев }}$ (если значения частных показателей качества принадлежат области адекватности с оптимальным радиусом по всем показателям). Все критерии качества ЦСУД обладают свойствами представительности, эластичности и простоты [5].

При прогнозировании процесса развития целостно-системного учебного действия применяем фактографический метод - метод наименьших квадратов (МНК). При этом анализируем процесс наращивания базисных компонентов ЦСУД относительно целостности и системности - ориентировки, исполнения и контроля через систему временного ряда. Поэтому в дальнейшем ЦСУД будет развиваться в соответствии с законом: $S=\sum_{i=1}^{n}\left(y_{i}^{*}-y_{i}\right)^{2} \Rightarrow \min$, где $y_{i}^{*}$ - расчетные значения исходного ряда, $y_{i}-$ фактические значения исходного ряда, $n$ - число наблюдений. $\mathrm{C}$ учётом адаптации к новым условиям необходимо ввести коррективы в прогнозные оценки развития ЦСУД через коэффициенты дисконтирования, которые характеризует изменение ценности информации во времени $\quad\left(\beta_{i} \leq 1\right)$ : $S=\sum_{i=1}^{n} \beta_{i}\left(y_{i}^{*}-y_{i}\right)^{2} \Rightarrow \min$. При прогнозной оценке устанавливается и дальность прогнозирования: $\tau=\frac{\Delta t}{t_{X}}$, где $\Delta t-$ абсолютное время упреждения, $t_{X}-$ величина эволюционного цикла развития ЦСУД [6].

$$
\pi\left(\sigma_{1}, \sigma_{2}, \ldots, \sigma_{n}\right)=\left(\pi_{1}\left(\sigma_{1}, \ldots, \sigma_{n}\right), \pi_{2}(\ldots), \ldots, \pi_{n}\left(\sigma_{1}, \ldots, \sigma_{n}\right)\right)
$$

Функцию $\pi\left(\sigma_{1}, \ldots, \sigma_{n}\right)$ на множестве всех возможных значений переменных $\sigma_{1}, \sigma_{2}, \ldots \sigma_{n}$ можно выразить в форме соотношения или в виде n-мерной таблицы n-векторов. Тогда формируем n-мерную таблицу нормальной формой игры $Г$.

Любая целостно-системная коммуникативная деятельность, как игра $Г$, разложима в некоторой позиции $\mathrm{X}$ относительно ориентировочного, исполнительного и контрольного компонентов, если не существует информационных множеств, которые содержали бы позиции из двух множеств одновременно: 1) $\mathrm{X}$ и все следующие за ней позиции; 2) остальные позиции дерева игры. В этом случае надо выделить подигру $\Gamma_{\mathrm{x}}$, состоящую из $\mathrm{X}$ всех следующих за ней позиций, и факторигру Г/X, состоящую из всех оставшихся позиций плюс $\mathrm{X}$,
В общем случае математическая модель целостно-системной деятельности (ЦСКД) коммуникативной обра3, соответствующий различным социальным уровням - от личностных до международных отношений, при которых происходит обмен двенадцатью $(\mathrm{n}=12)$ предметно-деятельностными отношениями. В зависимости от социальной ситуации субъекты коммуникативной деятельности, зная на различном уровне структуру целостносистемного цикла жизнедеятельности (ЦСЦЖ), применяют свои возможности относительно позиционных игр, их стратегии, нормальной формы игры и контролем процесса соответствия. При этом позиционная игра $\mathrm{n}$ лиц устанавливает топологическое дерево $Г$ с установленной вершиной A, начальной структуры игры, функцией выигрыша, которая устанавливает каждой финишной позиции дерева $\Gamma$ n-вектор, разделение структуры всех компаундных позиций дерева Г на $\mathrm{n}+1$ множеств $\mathrm{S}_{0}, \mathrm{~S}_{1}, \ldots, \mathrm{S}_{\mathrm{n}}$, - множества последовательности [7].

Стратегия игрока $i$, который воспроизводит структуру ЦСЦЖ, есть функция, которая устанавливает перенос каждому информационному множеству $S_{i}^{j}$ этого игрока некоторый индекс из $I_{i}^{j}$. Множество всех стратегий игрока $i$ есть сумма величин $\sum i$. Если результаты случайных действий известны в вероятностном отношении, то представляем функции выигрыша как математическое ожидание при условии, что игрок $i$ применяет стратегию $\sigma \in \sum i$ и применяем обозначение: многоуровневый

и функция выигрыша имеет вид: $\pi_{X}\left(\sigma_{1 \mid \Gamma_{X}}, \sigma_{2 \mid \Gamma_{X}}, \ldots, \sigma_{n \mid \Gamma_{X}}\right)_{[8] .}$

Целостно-системная коммуникативная деятельность может принимать форму антагонистической игры, если существует $\left(p_{1}, \ldots, p_{n}\right)$ нулевая сумма удовлетворяет условию $\sum_{i=1}^{n} p_{i}=0 . \quad$ Тогда $n$-компонента вектора выигрышей определяется остальными $n-1$ компонентами. В целом, нормальная форма конечной антагонистической игры приводится к матрице А с числом строк, равным числу действий игрока I, и с числом столбцов, равным числу действий игрока II.

При построении ориентировочного компонента ЦСКД возникают смешанные стратегии игрока как вероятностное распределение на множестве его чистых стратегий всех составляющих цикла. В этом 
случае, когда игрок имеет только конечное число $\mathrm{m}$ чистых стратегий, смешанная стратегия представляет собой т-вектор $\mathrm{x}=\left(\mathrm{x}_{1}, \ldots, \mathrm{x}_{\mathrm{m}}\right)$ ,удовлетворяющий условиям $x \geq 0$ и $\sum_{i=1}^{m} x_{i}=$ 1. Если обозначить множество всех смешанных стратегий игрока I через X, а множество всех смешанных стратегий игрока II через У, и предположить, что игроки I и II участвуют в матричной игре А, то если игрок I выбирает смешанную стратегию X, а игрок II выбирает У, то ожидаемый выигрыш будет равен

$$
A(x, y)=\sum_{i=1}^{m} \sum_{j=1}^{n} x_{i} a_{i j} y_{j}
$$

или в матричной форме: $A(x, y)=x A y^{T}$.

Моделирование исполнительного компонента ЦСКД связывается с разработкой стратегией поведения, которые устанавливают набор $\mathrm{N}$ вероятностных распределений и задают возможные альтернативы в каждой информационной коммуникации. При этом возникает множество распределений А, что ни одно распределение из А не предпочитается обоими субъектами ЦСКД другому распределению из А, но для любого не входящего в А распределения $((x, y) ;(a-x, b-y))$ в множестве А найдется распределение $\left(\left(x^{\prime}, y^{\prime}\right) ;(\mathrm{a}-\right.$ $\left.x^{\prime}, \mathrm{b}-y^{\prime}\right)$ ), которое устанавливают оба субъекта ЦСКД [9].

Моделирование контрольного компонента ЦСКД определяется вектором значений игры, который задаёт n-вектор $\varphi[\vartheta]$, удовлетворяющий аксиомам Шепли, выделяющих базисные условия математического моделирования целостно- системной коммуникативной деятельности педагогометрического анализа. Степень устойчивости ЦСКД задаёт норму поведения в форме коалиционной структуры в игре n-лиц. При этом возникает разбиение $\mathscr{T}=\left(T_{1}, T_{2}, \ldots, T_{m}\right)$ множества N. Данная структура представляет разбиение множества $\mathrm{N}$ на взаимно непересекающиеся коалиции. Возникающая конфигурацией задаётся парой $(x ; \mathscr{T})=\left(x_{1}, \ldots, x_{n} ; T_{1}, \ldots, T_{m}\right)$, где $\mathscr{T}$ коалиционная структура, а $x$ представляет собой n-вектор, удовлетворяющий условиям

$$
\sum_{i \in T_{k}} x_{i}=v\left(T_{k}\right)
$$

для $k=1, \ldots, m$. Реализация выделенных условий приводит к установлению индивидуальной рациональности, выражающей системный тип ориентировки в целостносистемной коммуникативной деятельности и её математической модели [10].

\section{Conclusion}

Развитие базисных показателей эрцгаммности математических моделей учебной деятельности формирующего исследования образовательных объектов относительно различных методов математического моделирования количественных отношений множества эрцгаммных параметров, позволяет создать адаптивные процессы математического моделирования подготовки широкопрофильных специалистов и их личностных качеств.

\section{References:}

1. Mishchik SA (2014) Pedagogometrika and mathematical modeling educational activity. Materialy Mezhdunarodnoy nauchnoy konferenctsii "Modern mathematics in science" - 30.06.2014. ISJ Theoretical \&Applied Science 6(14): 54-56 Caracas, Venezuela. doi: http://dx.doi.org/10.15863/TAS.2014.06.14.10

2. Mishchik SA (2014) Simulation training activity methods of mathematical logic. Materialy Mezhdunarodnoy nauchnoy konferenctsii "Eurapean Science and Education" - 30.07.2014. ISJ Theoretical \&Applied Science 6(15): 72-74 Marseille, France. doi: http://dx.doi.org/10.15863/TAS.2014.07.15.13

3. Mishchik SA (2014) Mathematical modeling system integrity-cycle of life activity - first goal pedagogometriki. Materialy Mezhdunarodnoy nauchnoy konferenctsii "European Applied Sciences" - 30.08.2014. ISJ Theoretical \&Applied Science 7(16): 77-79. Aix-en-Provence, France. doi: http://dx.doi.org/10.15863/TAS.2014.08.16.13

4. Mishchik SA (2014) Mathematical modeling system integrity-curricular activities - the second problem pedagogometriki. Materialy Mezhdunarodnoy nauchnoy konferenctsii "European Innovation" - 30.09.2014. ISJ Theoretical \&Applied Science 9(17): 126-128 Martigues, France. doi: http://dx.doi.org/10.15863/TAS.2014.09.17.21

5. Mishchik SA (2014) Mathematical modeling holistic-systemic communicative activity - the third task pedagogometriki. Materialy 


\begin{tabular}{l|lr|ll|ll} 
& ISRA (India) & $=\mathbf{1 . 3 4 4}$ & SIS (USA) & $=\mathbf{0 . 9 1 2}$ & ICV (Poland) & $=\mathbf{6 . 6 3 0}$ \\
Impact Factor: & ISI (Dubai, UAE) $=\mathbf{0 . 8 2 9}$ & PUHЦ (Russia) $=\mathbf{0 . 2 3 4}$ & PIF (India) & $=\mathbf{1 . 9 4 0}$ \\
& GIF (Australia) & $\mathbf{0 . 5 6 4}$ & ESJI (KZ) & $=\mathbf{1 . 0 4 2}$ & IBI (India) & $=\mathbf{4 . 2 6 0}$ \\
& JIF & $=\mathbf{1 . 5 0 0}$ & SJIF (Morocco) & $=\mathbf{2 . 0 3 1}$ & & \\
\hline
\end{tabular}

Mezhdunarodnoy nauchnoy konferenctsii "European Scientific Achievements" 30.10.2014. ISJ Theoretical \&Applied Science 10(18): 45-47 Brighton, UK. doi: http://dx.doi.org/10.15863/TAS.2014.10.18.11

6. Mishchik SA (2014) Mathematical modeling integrity - system performance subject - fourth task pedagogometriki. Materialy Mezhdunarodnoy nauchnoy konferenctsii "Eurapean Science and Technology" 30.11.2014. ISJ Theoretical \&Applied Science 11(19): 51-54 Southampton, UK. doi: http://dx.doi.org/10.15863/TAS.2014.11.19.10

7. Mishchik SA (2015) Pedagogometrik - science and academic subject. Materialy Mezhdunarodnoy nauchnoy konferenctsii "European Technology in Science" 28.02.2015. ISJ Theoretical \& Applied Science 02 (22): 103-106 Malmö, Sweden. doi: http://dx.doi.org/10.15863/TAS.2015.02.22.17

8. Tokmazov GV (2014) Matematicheskoe modelirovanie $\mathrm{v}$ uchebno-professional'noy deyatel'nosti. Materialy Mezhdunarodnoy nauchnoy konferentsii «Modern mathematics in science» - 30.06.2014. ISJ Theoretical \& Applied Science 6(14): 44-46. - Caracas, Venezuela. doi: http://dx.doi.org/10.15863/TAS.2014.06.14.8

9. Tokmazov GV (2014) Analysis says study skills in the study of mathematics, Materialy Mezhdunarodnoy nauchnoy konferenctsii "Eurapean Science and Education" 30.07.2014. ISJ Theoretical \&Applied Science 7(15): 72-74 Marseille, France. doi: http://dx.doi.org/10.15863/TAS.2014.07.15.9

10. Tokmazov GV (2014) Mathematical modeling research skills in educational activity methods of probability theory. Materialy Mezhdunarodnoy nauchnoy konferenctsii "Eurapean Science and Technology" 30.11.2014. ISJ Theoretical \&Applied Science 11(20): 66-69 Southampton, United Kingdom. doi:

http://dx.doi.org/10.15863/TAS.2014.11.19.13 